NSF-ITP-93-62

UCSBTH-93-14

hep-th/9305083

\title{
Four Dimensional Black Holes in String Theory
}

\author{
Steven B. Giddings \\ Joseph Polchinski \\ Andrew Strominger \\ Institute for Theoretical Physics \\ University of California \\ Santa Barbara, California 93106-4030 \\ and \\ Department of Physics \\ University of California \\ Santa Barbara, CA 93106-9530
}

\begin{abstract}
Exact solutions of heterotic string theory corresponding to fourdimensional charge $Q$ magnetic black holes are constructed as tensor products of an $S U(2) / Z(2 Q+2)$ WZW orbifold with a $(0,1)$ supersymmetric $S U(1,1) / U(1)$ WZW coset model. The spectrum is analyzed in some detail. "Bad" marginal operators are found which are argued to deform these theories to asymptotically flat black holes. Surprising behaviour is found for small values of $Q$, where low-energy field theory is inapplicable. At the minimal value $Q=1$, the theory degenerates. Renormalization group arguments are given that suggest the potential gravitational singularity of the low-energy field theory is resolved by a massive two-dimensional field theory. At $Q=0$, a stable, neutral "remnant," of potential relevance to the black hole information paradox, is found.
\end{abstract}




\section{Introduction}

One of our expectations of string theory is that as a quantum theory of gravity it should help us understand the puzzles of black holes, possibly by resolving the problem of curvature singularities. Perhaps a complete understanding of this must await a fully non-perturbative understand of the theory, but we have begun to investigate the role of singularities by studying exact classical string solutions. In particular, in [1] Witten found an exact conformal field theory corresponding to a two-dimensional dilatonic black hole. Although the stringy meaning of the singularity in this solution is not fully understood, it does serve as a well-defined example in which to address the questions. One concern, however, is that this two-dimensional example is oversimplified: singularities in higher-dimensional black holes may be qualitatively different. For this reason classical string solutions corresponding to four-dimensional black holes should also be useful. Such solutions have previously been found in [2, 3] as solutions of the low-energy effective theory for string theory. Although these charged solutions are known to leading order in the $\alpha^{\prime}$ expansion, corresponding exact solutions had not yet been found.

In this paper exact string solutions corresponding to certain limiting cases (in which the asymptotic two spheres have finite radius) of the magnetic black holes of ref. [2, 3] will be given. In these limiting cases the solutions become simple products of the black hole of [1] and a non-singular conformal field theory on a two sphere. The singularities found are therefore identical to those of the two-dimensional black holes. Further, the general solutions are expected to arise from these limiting cases by a deformation corresponding to a $(1,1)$ operator in the conformal field theory that we will construct. This suggests that even for the general solution the singularity is closely related to the two-dimensional version. Finally, it should be noted that such solutions may be relevant to the real world, if it is described by string theory. This

is seen by noting that for black holes whose radii are small compared to the dilaton Compton wavelength the dilaton is effectively massless. This means that real black holes below this scale could be described by the string solutions of this paper [4, 5, 6]. 
An important virtue of having the exact solutions is that it will allow us to study small values of the monopole charge, outside of the validity of the $\alpha^{\prime}$ expansion. We find some surprises for small values of the charge, namely two solutions with unexpected properties.

In the $\alpha^{\prime}$ expansion the balance between magnetic field and curvature gives the throat a radius proportional to the monopole charge, $r_{2}=Q$. In particular, there would not seem to be a neutral solution. In the exact solution this is corrected to $r_{2}=\left|Q^{2}-1\right|^{1 / 2}$, and so a $Q=0$ solution of finite radius exists. There is an extremal $Q=0$ solution with zero Hawking temperature, i.e. a neutral remnant. This is of obvious interest to advocates (among which we do not necessarily count ourselves!) of neutral remnants as a solution to the black hole information paradox.

Equally surprising is the fact that at $|Q|=1$ the radius is zero. The solution is trivial in the sense of conformal field theory, but that need not mean that it is uninteresting! There do not yet exist methods to analyze the interpolation between the exact throat and flat exterior solutions; this is one of the important open questions in this work. We will introduce an approximate picture, based on the renormalization group, which suggests that there exist solutions where the $Q=0, \pm 1$ throats do connect onto the flat exterior. In particular, at $|Q|=1$, the infinite throat narrows steadily to zero radius. This is perhaps a counterexample to the phenomenon of duality observed in toroidal compactification: down the throat, almost all states of the string move to arbitrarily high mass.

In this conjectured form of the $|Q|=1$ solution, the would-be singularity is resolved by a massive two-dimensional field theory. The nature of the singularity is essentially stringy, and does not have a spacetime interpretation. The generality of our arguments invite the speculation that this is a general method for resolving singularities in string theory.

The solutions factor into an $x t$ CFT, an angular CFT and an internal CFT. The $x t$ CFT is the linear dilaton or two-dimensional black hole, already known exactly. The angular 'monopole' CFT has a central charge which approaches 3 in the semiclassical limit. Rotational invariance implies the existence of an $S U(2)$ current algebra. Both of these observations point to a 
close connection with the WZW sigma model. In fact, we will find that the monopole theory is a coset of the level $k=2\left|Q^{2}-1\right| S U(2)$ WZW model by a discrete subgroup. For $Q>1$ this is

$$
\frac{S U(2)_{L} \times S U(2)_{R}}{Z(2 Q+2)_{R}} ;
$$

to make a $\hat{c}=4 \mathrm{CFT}$ the two-dimensional black hole factor would be the level $k^{\prime}=2\left(Q^{2}+1\right), S U(1,1) / U(1)$ coset theory.

In section 2 we review the low-energy effective field theory description of the magnetic black holes, and present the heterotic sigma model. In section 3 we bosonize the action and find that the result is indeed the coset (1.1) of the WZW model. We also describe a mild generalization, a bosonic theory with independent left- and right-moving monopole charges $Q_{L, R}$. In section 4 we bosonize the vertex operators. Taking appropriate monopole harmonic wavefunctions, the vertex operators are WZW vertex operators with a twist of the right-moving $U(1)$ boson. In section 5 we take a more abstract approach, looking for consistent level-matched string theories of the form (1.1), and recover the monopole theories constructed earlier.

In section 6 we return to the heterotic case, where $Q_{R}$ takes the value 1 appropriate for the spin connection. We verify world-sheet supersymmetry (which is $(0,2)$ due to a $U(1)$ symmetry), check level matching, and show that there is no spacetime supersymmetry. We show that the solutions are in general unstable if the monopole $U(1)$ is embedded in a non-Abelian group (as expected from low-energy considerations), but can be stable otherwise. We find the vertex operator corresponding to a widening of the throat toward the mouth, but are unable to extend the exact solution through the mouth to the asymptotic spacetime. Also, we verify that the exact solution is consistent with the index theorem for massless fermions in a monopole field. In section 7 we present the $Q=0$ solution, which is a slight variation of the $|Q|>1$ case. In section 8 we discuss $|Q|=1$. Our main tool is an approximate identification of the radial dependence of the solution with a renormalization group flow. The monopole CFT becomes strongly coupled and develops a mass gap in the region of the mouth, leaving a trivial CFT in the throat. The index theorem is useful in understanding the physics of the solution, and 
world-sheet instantons play an essential role. One of the main open problems is to obtain more control over the mouth region and verify that the existence of a solution interpolating between the known throat and asymptotic theories.

It is worth mentioning that the solution might have been presented in a different - and much shorter - manner. One could simply demonstrate that the coset model (1.1) obeys all the criteria for a building block of a consistent heterotic string theory: modular invariance, $(0,1)$ supersymmetry and a suitable GSO projection. The identification of (1.1) as a charge $Q$ monopole on $S_{2}$ then follows from the existence of $S U(2)$ rotational symmetries together with $2 Q$ massless spacetime fermions. We have instead chosen the scenic route to the final result, along which one views in detail the beautiful interplay between the current algebra, spacetime and sigma model descriptions of the theory. The insights gained via this route are important in our interpretation of the unexpected phenomona at $Q=0$ and $Q=1$.

There is by now an extensive literature on stringy black holes. An excellent review with references can be found in ref. [6].

\section{Review of Low-Energy Solutions}

The exact heterotic string solution that we will construct corresponds to the extremal member of a family of magnetically charged four-dimensional dilatonic black holes. These black holes first appeared [2, 3] as solutions of the the low-energy effective action

$$
S_{4} \propto \int d^{4} x \sqrt{-g} e^{-2 \Phi}\left(R+4(\nabla \Phi)^{2}-\frac{\alpha^{\prime}}{8} \operatorname{tr}\left(F^{2}\right)\right)
$$

which results when strings are compactified to four dimensions. We henceforth use units in which $\alpha^{\prime}=2$. The black hole solutions are parametrized by the sigma-model mass $M$, the charge $Q$, and the value of the dilaton at infinity, $\Phi_{0}$. For general values of these parameters they take the form

$$
\begin{aligned}
d s^{2} & =-4 Q^{2} \tanh ^{2} \sigma d t^{2}+\left(2 M+\Delta \sinh ^{2} \sigma\right)^{2}\left(4 d \sigma^{2}+d \Omega_{2}^{2}\right) \\
e^{2\left(\Phi-\Phi_{0}\right)} & =\frac{2 M+\Delta \sinh ^{2} \sigma}{\Delta \cosh ^{2} \sigma}
\end{aligned}
$$




$$
F=Q \epsilon_{2}
$$

where $d \Omega_{2}^{2}$ and $\epsilon_{2}$ are the line element and volume form on the unit two sphere, and we use a $U(1)$ embedding with $\operatorname{Tr}\left(F^{2}\right)=2 F_{\mu \nu} F^{\mu \nu}$. Here

$$
\Delta=2 M-\frac{Q^{2}}{2 M}
$$

To give a complete string solution eq. (2.2) should be supplemented by an internal solution corresponding to the compactification to four dimensions.

The spatial geometry of a constant $t$ slice through this solution is shown in Figure 1. In the limit $M \rightarrow Q / 2$ the throat length approaches infinity as $Q \ln (Q / \Delta)$. Near this limit there are four distinct regions,

i)

iv)

$$
\begin{array}{ll}
\sigma \gg \frac{1}{2} \ln (Q / \Delta) & \text { asymptotically flat region } \\
\sigma \sim \frac{1}{2} \ln (Q / \Delta) & \text { mouth } \\
\frac{1}{2} \ln (Q / \Delta) \gg \sigma \gg 1 & \text { throat } \\
\sigma \lesssim 1 & \text { black hole. }
\end{array}
$$

At the limit $M=Q / 2$ one then finds three distinct solution depending on where one's attention is focussed and how the dilaton is held fixed while taking the limit. The asymptotically flat region plus infinite throat is

$$
\begin{aligned}
d s^{2} & =-4 Q^{2} d t^{2}+\left(1+\frac{Q}{y}\right)^{2}\left(d y^{2}+y^{2} d \Omega^{2}\right) \\
e^{2\left(\Phi-\Phi_{0}\right)} & =1+\frac{Q}{y} \\
F & =Q \epsilon_{2}
\end{aligned}
$$

where $y=\Delta \cosh ^{2} \sigma$. If instead $\sigma=x+\sigma_{0}$ where $\frac{1}{2} \ln (Q / \Delta) \gg \sigma_{0} \gg 1$ the $M=Q / 2$ limit gives the throat solution:

$$
\begin{aligned}
d s^{2} & =-4 Q^{2} d t^{2}+4 Q^{2} d x^{2}+Q^{2} d \Omega_{2}^{2} \\
\Phi & =-x+\tilde{\Phi}_{0} \\
F & =Q \epsilon_{2} .
\end{aligned}
$$


Here the additive constant in the dilaton is shifted so that the dilaton is finite at finite $x$ rather than at infinity. Finally the black hole plus infinite throat is given by

$$
\begin{aligned}
d s^{2} & =-4 Q^{2} \tanh ^{2} \sigma d t^{2}+4 Q^{2} d \sigma^{2}+Q^{2} d \Omega_{2}^{2} \\
e^{2\left(\Phi-\hat{\Phi}_{0}\right)} & =\frac{Q}{\cosh ^{2} \sigma} \\
F & =Q \epsilon_{2}
\end{aligned}
$$

where once again the dilaton is shifted to be finite in the region of interest. Since the asymptotically flat region has disappeared in the latter two limits, it is not appropriate to associate the mass $M=Q / 2$ with the spacetime. An application of the ADM procedure to spacetimes of the form (2.7) which are asymptotic to (2.6) yields a mass proportional to $e^{2 \hat{\Phi}_{0}}$ [1].

In both of these latter cases the solutions are trivial products of two two-dimensional solutions. In both the angular solution is the round twosphere with constant radius threaded by a uniform magnetic flux. The other solution is in the first case the linear dilaton together with time, and in the second the two-dimensional black hole of [8, 1]. Both the linear dilaton vacuum and the black hole correspond to exact conformal field theories. A similar factorization occurs for analogous five-dimensional black holes in the extremal limit, with $S^{2}$ replaced by $S^{3}$ with torsion. In that case the angular theory corresponds to an exact conformal field theory and therefore the fivedimensional solution is an exact string solution [9, 10] when supplemented by an extra five dimensions. This together with the simplicity of the above $S^{2}$ theory leads to the conjecture that it also corresponds to an exact conformal field theory, and thus yields an exact string solution representing a fourdimensional black hole. The present paper will construct that theory.

A world-sheet description of such a solution is via a heterotic sigma model, with action $^{1}$

$$
S=\frac{1}{4 \pi} \int d^{2} z r_{2}^{2} G_{\mu \nu}^{S_{2}} \partial_{z} X^{\mu} \partial_{\bar{z}} X^{\nu}+\frac{1}{2 \pi} \int d^{2} z\left\{\bar{\lambda}_{R}\left(\partial_{\bar{z}}-i \omega_{\mu} \partial_{\bar{z}} X^{\mu}\right) \lambda_{R}\right.
$$

\footnotetext{
${ }^{1}$ Our conventions are $\alpha^{\prime}=2$ and $d^{2} z=2 d \sigma d \tau$. The current algebral fermion $\lambda_{L}$ has charge $e=1$. The action is well defined for $Q$ half-integer, but we will find below that $Q$ must in fact be an integer.
} 


$$
\left.\bar{\lambda}_{L}\left(\partial_{z}-i 2 Q A_{\mu}^{M} \partial_{z} X^{\mu}\right) \lambda_{L}+i 2 Q F_{\mu \nu}^{M} \psi_{R}^{\mu} \psi_{R}^{\nu} \bar{\lambda}_{L} \lambda_{L}\right\}
$$

Here $G_{\mu \nu}^{S^{2}}$ is the unit round metric on the two sphere, $\lambda_{L}$ is the current algebra fermion, $\lambda_{R}$ is the supersymmetric fermion in tangent space,

$$
\lambda_{R}=\left(e_{\mu}^{1}+i e_{\mu}^{2}\right) \psi_{R}^{\mu},
$$

$\omega_{\mu}$ is the spin connection for vectors on the two sphere, and $A_{\mu}^{M}$ is the gauge field of a magnetic monopole of unit charge. In the 'northern' coordinate patch the monopole potential is

$$
A_{\phi}^{M(N)}=\frac{1-\cos \theta}{2},
$$

and in the 'southern' patch it is

$$
A_{\phi}^{M(S)}=A_{\phi}^{M(N)}+i e^{-i \phi} \partial_{\phi} e^{i \phi}=-\frac{1+\cos \theta}{2} .
$$

The four fermion interaction is necessary for world-sheet supersymmetry. The one-loop beta-function equations fix the radius $r_{2}$ in terms of the charge $Q$

$$
r_{2}^{2}=Q^{2}
$$

The spin connection on the two-sphere is simply a monopole field of charge 1 , and the four-fermi interaction can be rewritten using

$$
\begin{aligned}
F_{\mu \nu}^{M} \psi_{R}^{\mu} \psi_{R}^{\nu} & =\frac{1}{2 r_{2}^{2}} \epsilon_{\mu \nu} \psi_{R}^{\mu} \psi_{R}^{\nu} \\
& =-\frac{i}{2 r_{2}^{2}} \bar{\lambda}_{R} \lambda_{R}
\end{aligned}
$$

The action then takes the form

$$
\begin{aligned}
S=\frac{1}{4 \pi} \int d^{2} z\left(G_{\mu \nu}\right. & \left.+B_{\mu \nu}\right) \partial_{z} X^{\mu} \partial_{\bar{z}} X^{\nu}+\frac{1}{2 \pi} \int d^{2} z\left\{\bar{\lambda}_{R}\left(\partial_{\bar{z}}-i A_{R \mu} \partial_{\bar{z}} X^{\mu}\right) \lambda_{R}\right. \\
& \left.+\bar{\lambda}_{L}\left(\partial_{z}-i A_{L \mu} \partial_{z} X^{\mu}\right) \lambda_{L}-h \bar{\lambda}_{R} \lambda_{R} \bar{\lambda}_{L} \lambda_{L}\right\}
\end{aligned}
$$


with backgrounds

$$
\begin{aligned}
G_{\mu \nu} & =r_{2}^{2} G_{\mu \nu}^{S_{2}} \\
B_{\mu \nu} & =0 \\
A_{R \mu} & =2 Q_{R} A_{\mu}^{M} \\
A_{L \mu} & =2 Q_{L} A_{\mu}^{M}
\end{aligned}
$$

and

$$
Q_{L}=Q, \quad Q_{R}=1,
$$

and a Thirring coupling, $h=-Q / r_{2}^{2}$. The world-sheet fermions in the north and south patches are related by

$$
\lambda_{R, L}^{(S)}=e^{-i 2 Q_{R, L} \phi} \lambda_{R, L}^{(N)},
$$

In order to make connection with the WZW model, we will bosonize the leftand right-moving fermons. The worldsheet supersymmetry will then not be explicit, and it will be just as easy to work with general charges $Q_{L, R}$. In section 6 we will return to the supersymmetric case $Q_{R}=1$.

\section{Equivalence to WZW Action}

We now bosonize (2.14), introducing a third embedding field $X^{3}$ which is periodic with period $2 \pi$. The bosonized action is

$$
\begin{aligned}
S= & \frac{1}{4 \pi} \int d^{2} z\left\{r_{2}^{2} G_{\mu \nu}^{S_{2}} \partial_{z} X^{\mu} \partial_{\bar{z}} X^{\nu}+r_{1}^{2}\left(\partial_{z} X^{3}-A_{\mu}^{+} \partial_{z} X^{\mu}\right)\left(\partial_{\bar{z}} X^{3}-A_{\mu}^{+} \partial_{\bar{z}} X^{\mu}\right)\right. \\
& \left.+A_{\mu}^{-}\left(\partial_{z} X^{3} \partial_{\bar{z}} X^{\mu}-\partial_{\bar{z}} X^{3} \partial_{z} X^{\mu}\right)+\frac{1}{2} A_{\mu}^{-} A_{\nu}^{+}\left(\partial_{z} X^{\mu} \partial_{\bar{z}} X^{\nu}-\partial_{z} X^{\nu} \partial_{\bar{z}} X^{\mu}\right)\right\}
\end{aligned}
$$

where

$$
r_{1}^{2}=1+2 h
$$

and

$$
A_{\mu}^{ \pm}=A_{\mu}^{L} \pm A_{\mu}^{R}=2 Q_{ \pm} A_{\mu}^{M}, \quad Q_{ \pm}=Q_{L} \pm Q_{R}
$$


The invariances

$$
\begin{aligned}
X^{3} & \rightarrow X^{3}+\epsilon^{L}\left(X^{\mu}\right)+\epsilon^{R}\left(X^{\mu}\right) \\
A_{\mu}^{L} & \rightarrow A_{\mu}^{L}+\partial_{\mu} \epsilon^{L} \\
A_{\mu}^{R} & \rightarrow A_{\mu}^{R}+\partial_{\mu} \epsilon^{R} \\
B_{\mu \nu} & \rightarrow B_{\mu \nu}+\epsilon^{L} F_{\mu \nu}^{L}-\epsilon^{R} F_{\mu \nu}^{R}
\end{aligned}
$$

provide a check that the gauge field has been correctly introduced.円 Notice in particular that the $A_{\mu}^{-}$term must have an $h$-independent coefficient because it corresponds to torsion, which is quantized. Similarly, the two terms in $\partial_{z} X^{3}+A_{\mu}^{+} \partial_{z} X^{\mu}$ must have $h$-independent relative coefficient in order that the mapping between the north and south coordinate patches respect the $2 \pi$ periodicity of $X^{3}$.

The quantization of the torsion is seen explicitly if one integrates by parts,

$$
-\frac{1}{4 \pi} \int d^{2} z X^{3} F_{\mu \nu}^{-} \partial_{z} X^{\mu} \partial_{\bar{z}} X^{\nu}
$$

Under $X^{3} \rightarrow X^{3}+2 \pi$, this changes by

$$
-\frac{i}{4} \int F_{\mu \nu}^{-} d X^{\mu} d X^{\nu}=-i 2 \pi n Q_{-}
$$

where $n$ is the winding number of the map from the world-sheet to $S_{2}$. The path integral is therefore well-defined for all $n$ if $Q_{-}$is integer. The restriction to integer values is due to a global world-sheet anomaly. In the fermionic language, if we take a genus zero world-sheet mapped once to the spacetime two-sphere, there are $2 Q_{-}$net fermionic zero modes; this number must be even. There is also a spacetime interpretation: in the left-moving Ramond sector, the charges are half-integer.

The three-dimensional target space of the bosonized theory has curvatures

$$
\begin{aligned}
& R_{\hat{1} \hat{1}}=R_{\hat{2} \hat{2}}=\frac{2 r_{2}^{2}-r_{1}^{2} Q_{+}^{2}}{2 r_{2}^{4}} \\
& R_{\hat{3} \hat{3}}=\frac{r_{1}^{2} Q_{+}^{2}}{2 r_{2}^{4}}
\end{aligned}
$$

\footnotetext{
${ }^{1}$ The last term in the action vanishes for the particular background (3.3), but was included to allow independent $L$ and $R$ gauge transformations.
} 
and torsion

$$
H_{\hat{1} \hat{2} \hat{3}}=-Q_{-} / r_{2}^{2} r_{1}
$$

where we have for convenience used tangent space indices

$$
e_{\hat{\imath}}=\left(r_{2} d \theta, r_{2} \sin \theta d \phi, r_{1}\left(d X^{3}-A_{\phi}^{+} d \phi\right)\right) .
$$

The one-loop beta functions are then

$$
\begin{aligned}
\mu \partial_{\mu} \ln r_{2} & =R_{\hat{1} \hat{1}}-\frac{1}{2} H_{\hat{1} \hat{2} \hat{3}}^{2} \\
& =\frac{2 r_{2}^{2} r_{1}^{2}-r_{1}^{4} Q_{+}^{2}-Q_{-}^{2}}{2 r_{2}^{4} r_{1}^{2}} \\
\mu \partial_{\mu} \ln r_{1} & =R_{\hat{3} \hat{3}}-\frac{1}{2} H_{\hat{1} \hat{2} \hat{3}}^{2} \\
& =\frac{r_{1}^{4} Q_{+}^{2}-Q_{-}^{2}}{2 r_{2}^{4} r_{1}^{2}} .
\end{aligned}
$$

By redefining $\lambda_{R} \leftrightarrow \bar{\lambda}_{R}, \lambda_{L} \leftrightarrow \bar{\lambda}_{L}$, and $z \leftrightarrow \bar{z}$, we may assume

$$
Q_{L} \geq Q_{R} \geq 0
$$

The one-loop beta functions then vanish for

$$
r_{2}=\sqrt{Q_{+} Q_{-}}, \quad r_{1}=\frac{r_{2}}{Q_{+}}=\sqrt{\frac{Q_{-}}{Q_{+}}} .
$$

This also implies for the Thirring coupling

$$
h=-\frac{Q_{R}}{Q_{+}} .
$$

The action is now

$$
\begin{aligned}
S=\frac{Q_{+} Q_{-}}{4 \pi} \int d^{2} z\left\{\left(G_{\mu \nu}^{S_{2}}\right.\right. & \left.+4 A_{\mu}^{M} A_{\nu}^{M}\right) \partial_{z} X^{\mu} \partial_{\bar{z}} X^{\nu} \\
& \left.+\frac{1}{Q_{+}^{2}}\left(\partial_{z} X^{3}-4 Q_{+} A_{\mu}^{M} \partial_{z} X^{\mu}\right) \partial_{\bar{z}} X^{3}\right\}
\end{aligned}
$$


As we have discussed in the introduction, one expects a close relation to the SU(2) WZW model 11]. Topologically, the sigma model (3.14) is an $S_{1}$ bundle over $S_{2}$, with winding number $2 Q_{+}$and torsion $\frac{1}{8 \pi^{2}} \int H=Q_{-}$. The $S U(2)$ group manifold is also an $S_{1}$ bundle over $S_{2}$ (the Hopf fibration). Writing the group element in terms of Euler angles

$$
g=e^{i \phi \sigma_{3} / 2} e^{i \theta \sigma_{2} / 2} e^{i(\xi-\phi) \sigma_{3} / 2}
$$

with ranges

$$
0 \leq \theta \leq \pi, \quad 0 \leq \phi \leq 2 \pi, \quad 0 \leq \xi \leq 4 \pi,
$$

the fiber coordinate is $\xi$ and the base coordinates $\theta, \phi$. One sees from eq. (3.15) that at the north pole $(\theta=0), \xi$ is a good coordinate, while at the south pole $(\theta=\pi), \xi-2 \phi$ is a good coordinate. Noticing the ranges, the winding number is one. Thus, if we have an SU(2) WZW model of torsion (level)

$$
k=2 Q_{+} Q_{-},
$$

and if we identify

$$
\xi=\frac{1}{Q_{+}} X^{3}, \quad \xi \sim \xi+\frac{2 \pi}{Q_{+}},
$$

the group manifold is is topologically the same as the sigma model (3.14). Not surprisingly, one finds that in terms of Euler angles the WZW action[11 is precisely eq. (3.14). The exact central charge is then

$$
c=\frac{3 k}{k+2} .
$$

The monopole model (2.14) is invariant under $S U(2)$ spatial rotations and under one non-anomalous linear combination of chiral fermion rotations, $Q_{L} \epsilon_{L}=Q_{R} \epsilon_{R}$. Correspondingly, the WZW model with identification (3.18) is invariant under $S U(2)$ left-multiplication and $U(1)$ right-multiplication, the remainder of the right $S U(2)$ being inconsistent with the identification. The Noether current of the $U(1), \xi \rightarrow \xi+\epsilon$, is

$$
\begin{aligned}
& j_{N z}=\frac{i Q_{-}}{2}\left(\partial_{z} X^{3}-4 Q_{+} A_{\mu}^{M} \partial_{z} X^{\mu}\right) \\
& j_{N \bar{z}}=\frac{i Q_{-}}{2} \partial_{\bar{z}} X^{3}
\end{aligned}
$$


This current is not chiral, but becomes so after a trivial redefinition $\left(j_{z}, j_{\bar{z}}\right)=$ $\left(j_{N z}, j_{N \bar{z}}\right)+i Q_{-}\left(\partial_{z} X^{3},-\partial_{\bar{z}} X^{3}\right) / 2$,

$$
\begin{aligned}
& j_{z}=i Q_{-}\left(\partial_{z} X^{3}-2 Q_{+} A_{\mu}^{M} \partial_{z} X^{\mu}\right)=\frac{k}{2} \operatorname{tr}\left(\sigma^{3} g^{-1} \partial_{z} g\right) \\
& j_{\bar{z}}=0 .
\end{aligned}
$$

From the $S U(2)$ current algebra, we have

$$
j_{z}(z) j_{z}(0) \sim \frac{k}{2 z^{2}} .
$$

We can write $j_{z}$ as the gradient of an analytic scalar,

$$
j_{z}(z)=\frac{i k}{2} \partial_{z} \xi_{R},
$$

with

$$
\xi_{R}(z) \xi_{R}(0) \sim-\frac{2}{k} \ln z .
$$

The normalization is fixed so that $j_{z}(z) \xi_{R}(0)=-i / z$. Notice that $\xi_{L}=\xi-\xi_{R}$ is not antianalytic, and $\partial_{\bar{z}} \xi_{L}$ is not a conserved current.

In summary, the monopole CFT is

$$
\frac{S U(2)_{L} \times S U(2)_{R}}{Z\left(2 Q_{+}\right)_{R}} .
$$

with the unbroken $S U(2)$ currents being antianalytic and the unbroken $U(1)$ being analytic. In our conventions right-moving (analytic) happens to coincide with right-multiplication. The $z-\bar{z}$ asymmetry of the construction arises from the inequality $Q_{R}<Q_{L}$. For $Q_{R}=Q_{L}$, the level $k$ is zero and the CFT is trivial; we will discuss this case further in section 8 .

\section{The Spectrum}

To complete the identification, we will express the vertex operators of the monopole theory in terms of the WZW operators. The fermions $\lambda_{R, L}$ have 
momenta $p_{3}=\frac{1}{2}$ and winding numbers $w_{3}= \pm 1$ in the 3 -direction. The right- and left- fermion numbers are then

$$
F_{R}=p_{3}+\frac{w_{3}}{2}, \quad F_{L}=p_{3}-\frac{w_{3}}{2} .
$$

Note for future reference that $F_{L}$ is the same as $U(1)$ charge $e$. The canonical bosonization formula is 12

$$
\mathcal{O}_{F_{R}, F_{L}}(0)=\exp \left\{i \frac{F_{R}+F_{L}}{2} X^{3}(0)+2 \pi i\left(F_{R}-F_{L}\right) \int_{0}^{\infty} d \sigma \pi_{3}(\sigma)\right\} .
$$

The canonical momentum is

$$
\begin{aligned}
\pi_{3} & =\frac{Q_{-}}{4 \pi Q_{+}}\left(\partial_{\bar{z}} X^{3}-\partial_{z} X^{3}+4 Q_{+} A_{\mu}^{M} \partial_{z} X^{\mu}\right) \\
& =\frac{Q_{-}}{4 \pi} \partial_{\sigma}\left(\xi_{L}-\xi_{R}\right)
\end{aligned}
$$

where $\xi_{R, L}$ are as defined at the end of the previous section. The bosonization formula is then

$$
\mathcal{O}_{F_{R}, F_{L}}=\exp \left\{i\left(F_{L} Q_{L}+F_{R} Q_{R}\right) \xi_{L}+i\left(F_{L} Q_{R}+F_{R} Q_{L}\right) \xi_{R}\right\}
$$

In particular,

$$
\begin{aligned}
& \lambda_{R}=\exp \left\{i\left(Q_{R} \xi_{L}+Q_{L} \xi_{R}\right)\right\} \\
& \lambda_{L}=\exp \left\{i\left(Q_{L} \xi_{L}+Q_{R} \xi_{R}\right)\right\}
\end{aligned}
$$

This is the same form that holds without the gauge interaction of the fermions (but with the Thirring coupling $(3.13)$ ), though in the present case $\xi_{L}$ is not a free field.

We now wish to relate the vertex operators of the monopole theory into those of the exact CFT. A vertex operator is a polynomial in fields and derivatives, times a wavefunction of the embedding coordinates $X^{\mu}$. There is an important subtlety: the charged fields are not globally defined. For example, from eq. (2.17) we see that at the south pole $\lambda_{R, L}^{(N)}$ is $e^{2 i Q_{R, L} \phi}$ times 
a single-valued function. In order to make a conformally invariant vertex operator, we need a compensating singularity in the wavefunction.

The necessary wavefunctions are the monopole harmonics, which we briefly review 13. We start by recalling the relation between the ordinary spherical harmonics and the representation matrices of $S U(2)$. Consider first a particle moving on the $S U(2)$ group manifold. The representation matrices

$$
D_{m_{L}, m_{R}}^{j}(g)
$$

form a complete set of wavefunctions on $S U(2)$. These transform as spin- $j$ under both left- and right-multiplication,

$$
D_{m_{L}, m_{R}}^{j}\left(g_{L}^{-1} g g_{R}\right)=D_{m_{L}, m_{L}^{\prime}}^{j}\left(g_{L}^{-1}\right) D_{m_{L}^{\prime}, m_{R}^{\prime}}^{j}(g) D_{m_{R}^{\prime}, m_{R}}^{j}\left(g_{R}\right)
$$

Now let us consider the two-sphere $S_{2}$ regarded as a coset $S U(2) / U(1)_{R}$. We obtain a complete set of wavefunctions by restricting the set (4.6) to those which are invariant under the identification-namely, those with $m_{R}=0$. Left $S U(2)$ takes this set into itself, and is the rotational symmetry of the twosphere. The transformation law (4.7) thus identifies $D_{m, 0}^{j}(g)$ as the spherical harmonic $Y_{m}^{j}$, up to normalization.

Now we consider $m_{R}=q / 2 \neq 0$. The representation matrix (4.6) is no longer well-defined on the coset $S_{2}$, so let us make a convenient choice of of $\operatorname{map} S_{2} \rightarrow S U(2)$

$$
g_{\theta, \phi}=e^{i \phi \sigma_{3} / 2} e^{i \theta \sigma_{2} / 2} e^{-i \phi \sigma_{3} / 2} .
$$

With this choice, the elements

$$
D_{m, q / 2}^{j}\left(g_{\theta, \phi}\right)
$$

are well-behaved at the north pole but not at the south pole. Note that the $\phi$-dependence of $D_{m, q / 2}^{j}\left(g_{\theta, \phi}\right)$ is $e^{i(m-q / 2) \phi}$. At $\theta=0$, the only nonvanishing matrix element is $m=q / 2$, which is single-valued. At $\theta=\pi$, however, the only nonvanishing element is $m=-q / 2$, which thus is multi-valued as $e^{-i q \phi}$. Thus, $D_{m, q / 2}^{j}\left(g_{\theta, \phi}\right)$ is the wavefunction of a particle of unit charge in a monopole field of strength $-q / 2$. 
Consider also the rotational properties of these functions. Expanding in Euler angles, we can write

$$
g_{L}^{-1} g_{\theta, \phi}=g_{\theta^{\prime}, \phi^{\prime}} e^{i \xi^{\prime} \sigma_{3} / 2}
$$

for some functions $\theta^{\prime}\left(\theta, \phi, g_{L}\right), \phi^{\prime}\left(\theta, \phi, g_{L}\right), \xi^{\prime}\left(\theta, \phi, g_{L}\right)$. This defines the action of $S U(2)_{L}$ on the coset $S_{2},(\theta, \phi) \rightarrow\left(\theta^{\prime}, \phi^{\prime}\right)$. Then the transformation (4.7) becomes

$$
D_{m, q / 2}^{j}\left(g_{\theta^{\prime}, \phi^{\prime}}\right)=D_{m, q / 2}^{j}\left(g_{L}^{-1} g_{\theta, \phi} e^{-i \xi^{\prime} \sigma_{3} / 2}\right)=D_{m, m^{\prime}}^{j}\left(g_{L}^{-1}\right) D_{m^{\prime}, q / 2}^{j}\left(g_{\theta, \phi}\right) e^{-i \xi^{\prime} q / 2}
$$

This is the spin- $j$ transformation up to a phase. The phase, which is independent of the state $j, m$, is just the gauge transformation needed to bring the Dirac string back to the south pole after a rotation. Notice, by the way, that there is a lower bound on the angular momentum

$$
j \geq \frac{q}{2} \text {. }
$$

Let us also note the property

$$
-\nabla_{-q}^{2} D_{m, q / 2}^{j}\left(g_{\theta, \phi}\right)=\left\{j(j+1)-q^{2} / 4\right\} D_{m, q / 2}^{j}\left(g_{\theta, \phi}\right),
$$

where $\nabla_{-q}^{2}$ is the covariant Laplacian for a particle of unit charge in a monopole field $-q / 2$.

We now make vertex operators by combining $\mathcal{O}_{F_{R}, F_{L}}$ with an appropriate wavefunction. Under the gauge symmetry (3.4),

$$
\delta \mathcal{O}_{F_{R}, F_{L}}=i\left(F_{L} \epsilon^{L}+F_{R} \epsilon^{R}\right) \mathcal{O}_{F_{R}, F_{L}}
$$

The total monopole field felt by $\mathcal{O}_{F_{R}, F_{L}}$ is then

$$
q=2 F_{L} Q_{L}+2 F_{R} Q_{R}
$$

The proper vertex operators are therefore

$$
\begin{aligned}
D_{m, q / 2}^{j}\left(g_{\theta, \phi}\right) \mathcal{O}_{F_{R}, F_{L}} & =D_{m, q / 2}^{j}\left(g_{\theta, \phi}\right) \exp \left\{i q \xi / 2+i\left(F_{R}-F_{L}\right) Q_{-} \xi_{R}\right\} \\
& =D_{m, q / 2}^{j}(g) \exp \left\{i\left(F_{R}-F_{L}\right) Q_{-} \xi_{R}\right\} .
\end{aligned}
$$


The translation between the wavefunctions (4.6) on $S U(2)$ and the current algebra primary fields is known[14],

$$
D_{m_{L}, m_{R}}^{j}(g(z, \bar{z}))=\tilde{\phi}_{m_{L}}^{j}(\bar{z}) \phi_{m_{R}}^{j}(z)
$$

so the final result is

$$
D_{m, q / 2}^{j}\left(g_{\theta, \phi}\right) \mathcal{O}_{F_{R}, F_{L}}(z, \bar{z})=\tilde{\phi}_{m}^{j}(\bar{z}) \phi_{q / 2}^{j}(z) \exp \left\{i\left(F_{R}-F_{L}\right) Q_{-} \xi_{R}(z)\right\} .
$$

This is a satisfying result: consistent with the identification (3.25) of the theory as a coset, the vertex operators are current algebra fields with a twist of $U(1)_{R}$. Also, separating the analytic primary into a parafermionic primary and a free boson part,

$$
\phi_{m}^{j}(z)=\psi_{m}^{j}(z) e^{i m \xi_{R}(z)}
$$

we have

$$
D_{m, q / 2}^{j}\left(g_{\theta, \phi}\right) \mathcal{O}_{F_{R}, F_{L}}(z, \bar{z})=\tilde{\phi}_{m}^{j}(\bar{z}) \psi_{q / 2}^{j}(z) \exp \left\{i\left(F_{L} Q_{R}+F_{R} Q_{L}\right) \xi_{R}(z)\right\} .
$$

The energy momentum tensor is

$$
T_{z z}=\frac{1}{2(k+2)} j_{z}^{a} j_{z}^{a}, \quad T_{\bar{z} \bar{z}}=\frac{1}{2(k+2)} j_{\bar{z}}^{a} j_{\bar{z}}^{a} .
$$

We can also decompose into a parafermion part and a free boson part,

$$
T_{z z}(z)=T_{z z}^{S U(2) / U(1)}(z)-\frac{k}{4} \partial_{z} \xi \partial_{z} \xi
$$

The states (4.20) thus have weights

$$
\begin{aligned}
\tilde{L}_{0} & =\frac{j(j+1)}{k+2} \\
L_{0} & =\frac{j(j+1)}{k+2}-\frac{q^{2}}{4 k}+\frac{\left(F_{R} Q_{L}+F_{L} Q_{R}\right)^{2}}{k} \\
& =\tilde{L}_{0}+\frac{F_{R}^{2}-F_{L}^{2}}{2} .
\end{aligned}
$$


Let us check the correspondence with the semiclassical expansion for some low-lying states. The tachyon has $F_{R}=F_{L}=0$, so the vertex operators are just the spherical harmonics $D_{m, 0}^{j}(g)$. Using the one loop result $k=2 r_{2}^{2}$, the dimension is

$$
L_{0}+\tilde{L}_{0}=\frac{j(j+1)}{r_{2}^{2}+1},
$$

while the spin $L_{0}-\tilde{L}_{0}=0$. This matches the semiclassical value for $r_{2}>>1$, the ' +1 ' being a correction to the semiclassical result. The vertex operator with one $\lambda_{R}$ oscillator excited is

$$
D_{m, Q_{R}}^{j}\left(g_{\theta, \phi}\right) \lambda_{R}=\tilde{\phi}_{m}^{j}(\bar{z}) \phi_{Q_{R}}^{j}(z) \exp \left\{i Q_{-} \xi_{R}(z)\right\}
$$

The spin is $L_{0}-\tilde{L}_{0}=\frac{1}{2}$, while the dimension is

$$
\begin{aligned}
L_{0}+\tilde{L}_{0} & =\frac{j(j+1)}{r_{2}^{2}+1}+\frac{1}{2} \\
& =\frac{j(j+1)-Q_{R}^{2}}{r_{2}^{2}+1}+\frac{1}{2}+\frac{h^{2}}{1+2 h} \frac{r_{2}^{2}}{r_{2}^{2}+1} .
\end{aligned}
$$

In the last line, the first term is from the monopole Laplacian (4.13), while the last is the shift in the dimension of $\lambda$ from the Thirring interaction (we have used eq. (3.13) for the Thirring coupling). Again the "+1" represents corrections to the semiclassical result. Similarly, the vertex operator with one $\lambda_{L}$ is

$$
D_{m, Q_{L}}^{j}\left(g_{\theta, \phi}\right) \lambda_{L}=\tilde{\phi}_{m}^{j}(\bar{z}) \phi_{Q_{L}}^{j}(z) \exp \left\{-i Q_{-} \xi_{R}(z)\right\} .
$$

The spin is $L_{0}-\tilde{L}_{0}=-\frac{1}{2}$, and the dimension is

$$
\begin{aligned}
L_{0}+\tilde{L}_{0} & =\frac{j(j+1)}{r_{2}^{2}+1}-\frac{1}{2} \\
& =\frac{j(j+1)-Q_{L}^{2}}{r_{2}^{2}+1}+\frac{1}{2} \frac{r_{2}^{2}-1}{r_{2}^{2}+1}+\frac{h^{2}}{1+2 h} \frac{r_{2}^{2}}{r_{2}^{2}+1},
\end{aligned}
$$

with the semiclassical limit again evident. 


\section{Level Matching}

Now let us make a new beginning, from a different point of view. We start with a level $k S U(2)$ WZW theory, and without reference to any spacetime interpretation we try to make a consistent string theory by twisting on $Z(N)_{R}$ for some integer $N$ :

$$
\frac{S U(2)_{L} \times S U(2)_{R}}{Z(N)_{R}} .
$$

A general twisted vertex operator is of the form

$$
\left(\tilde{\jmath}_{\tilde{K}} \cdot \tilde{\phi}_{m_{L}}^{j}\right)\left(j_{K} \cdot \phi_{m_{R}}^{j}\right) e^{i w_{3} k \xi_{R} / N} .
$$

Here $w_{3}$, which runs from 0 to $N-1$, is the winding number associated to the right moving $U(1)$ boson $\xi_{R}$. That is, $\xi_{R}$ shifts by $4 \pi w_{3} / N$ as $z$ encircles 0 :

$$
\xi_{R}(z) e^{i w_{3} k \xi_{R}(0) / N} \sim-\frac{2 i w_{3} \ln z}{N} e^{i w_{3} k \xi_{R}(0) / N} .
$$

The notations $j_{K}$ and $\tilde{j}_{\tilde{K}}$ stand for generic products of raising operators $j_{-n}^{a}$ and $\tilde{j}_{-n}^{a}$ respectively.t The untwisted part of (5.2) depends on $\xi_{R}$ as $e^{i J_{3} \xi_{R}}$ and the full operator as $e^{i J_{3}^{\prime} \xi_{R}}$, where

$$
J_{3}=m_{R}+N_{+}-N_{-}, \quad J_{3}^{\prime}=J_{3}+\frac{w_{3} k}{N},
$$

$N_{ \pm}$being the number of $j^{ \pm}$raising operators. The weights are therefore

$$
\begin{aligned}
L_{0} & =\frac{j(j+1)}{k+2}+|K|+\frac{J_{3}^{\prime 2}-J_{3}^{2}}{k} \\
\tilde{L}_{0} & =\frac{j(j+1)}{k+2}+|\tilde{K}|,
\end{aligned}
$$

where $|\tilde{K}|,|K|$ are the total levels of the raising operators.

\footnotetext{
${ }^{1}$ In eq. (5.2) we have acted first with the raising operators and then twisted. Alternately, we can first twist the primary, and then act with the twisted algebra of $j_{-n}^{3}, j_{-n \mp 2 w / N}^{ \pm}$, and $\tilde{\jmath}_{-n}^{a}$. These descriptions are equivalent.
} 
A consistent string theory requires level matching. The level mismatch here is

$$
\begin{aligned}
L_{0}-\tilde{L}_{0} & =\frac{J_{3}^{\prime 2}}{k}-\frac{J_{3}^{2}}{k} \bmod \mathbf{Z} \\
& =\frac{w_{3}}{N}\left(2 J_{3}+\frac{w_{3} k}{N}\right) \bmod \mathbf{Z}
\end{aligned}
$$

Let us for the present section think of this CFT as a background in bosonic string theory. The CFT is then modular invariant by itself, so in particular the spectrum must be restricted to states for which $L_{0}-\tilde{L}_{0}$ is an integer. Let us focus on $w_{3}=1$; by assumption there are states with $w_{3}=1$, or we would redefine $N \rightarrow N /\left(w_{3}\right)_{\min }$. Then

$$
2 J_{3}+\frac{k}{N} \in N \mathbf{Z}
$$

Since $2 J_{3}$ is an integer, we learn that $k / N$ must be an integer, $N^{\prime}$. This is the quantization of the torsion on the coset manifold. The level matching condition is then

$$
2 J_{3}+N^{\prime} \in N \mathbf{Z}, \quad\left(w_{3}=1\right),
$$

which has solutions. Since $J_{3}$ and $w_{3}$ are both conserved, the $w_{3}=1$ result plus closure of the OPE implies that at general $w_{3}$

$$
2 J_{3}+w_{3} N^{\prime} \in N \mathbf{Z},
$$

or equivalently

$$
J_{3}+J_{3}^{\prime} \in N \mathbf{Z}, \quad J_{3}-J_{3}^{\prime} \in N^{\prime} \mathbf{Z}
$$

These states satisfy level matching, and a check of the characters shows that the partition function is in fact modular-invariant.

Now let us relate these theories to the monopole background. Comparing the twisting and the level gives

$$
N=2 Q_{+}, \quad N^{\prime}=Q_{-} .
$$

Also, comparing the $\xi_{R}$ dependences of the vertex operators (4.20) and (5.2), we have

$$
F_{R}+F_{L}=2 \frac{J_{3}+J_{3}^{\prime}}{N}, \quad F_{R}-F_{L}=\frac{J_{3}^{\prime}-J_{3}}{N^{\prime}}
$$


The condition (5.10) is thus that the total right plus left fermion number be even, which is the diagonal GSO projection. Note that the modular-invariant spectrum necessarily includes the Ramond-Ramond sectors with half-integer $F_{R, L}$.

Notice that eq. (5.11) implies that $Q_{-}$is an integer, as we found in the previous section, but allows $Q_{+}$to be half-integer. In this case

$$
Q_{L}=\frac{N+2 N^{\prime}}{4}, \quad Q_{R}=\frac{N-2 N^{\prime}}{4}
$$

are not half-integer, and the fields $\lambda_{L, R}$ have gauge-invariant Dirac string singularities. There is a simple reason why these theories are consistent: the actual states appearing in the GSO-projected spectrum all have properly quantized charge. Notice also that when $N$ is even the interchange

$$
N \leftrightarrow 2 N^{\prime}
$$

just flips the sign of $Q_{R}$. This is equivalent to the original theory, with redefinition $\lambda_{R} \rightarrow \bar{\lambda}_{R}$. In the bosonized form this is a duality transformation on $\xi$.

This completes our construction of the monopole CFT for bosonic string theory. We conclude with one general remark. Twisting by $Z(N)_{R}$, one might naively restrict the spectrum to $2 J_{3}^{\prime} \in N \mathbf{Z}$. This would differ from the actual spectrum (5.10) by a term proportional to winding number. It is well known that in twisting (orbifolding), it is not in general possible to determine the projection naively; rather, one must explicitly solve the levelmatching condition. We would like to point out that this is a special case of a well-known ambiguity in field theory: one always is free to add to a Noether current a term which is trivially conserved, $j^{a} \rightarrow j^{a}+\partial_{b} K^{a b}$ for any antisymmetric $K^{a b}$, as we have done in going from the current (3.20) to the current (3.21). In a topologically nontrivial sector, this changes the conserved charge. In the present case, there is a simple interpretation for the actual projection. The charge $J_{3}^{\prime}$ corresponds (up to normalization) to the chiral current (3.21), which one can think of as generating

$$
\delta \xi_{R}=\epsilon, \quad \delta \xi_{L}=0
$$


while $\frac{1}{2}\left(J_{3}+J_{3}^{\prime}\right)$ corresponds to the naive Noether current (3.20) and generates

$$
\delta \xi_{R}=\frac{\epsilon}{2}, \quad \delta \xi_{L}=\frac{\epsilon}{2}
$$

In a non-winding sector, only the combination $\xi_{R}+\xi_{L}$ appears, and these symmetries are equivalent, but in winding sectors they differ. Level matching picks out states invariant under the vector-like symmetry (5.16).

\section{Heterotic String Background}

As discussed in section 2, the heterotic string in a monopole background of charge $Q$ is a special case of the action (2.14), with

$$
Q_{L}=Q, \quad Q_{R}=1,
$$

and a particular value of the Thirring interaction. Since we found that in the bosonic case the Thirring coupling was fixed by conformal invariance, it must be that for $Q_{R}=1$ the theory is actually superconformally invariant.

Let us show this, following the general approach of the previous section. We will not initially assume $Q_{R}=1$. It is well-known that a twist of the $S U(2)$ current algebra produces an $N=2$ supersymmetry algebra:

$$
\begin{aligned}
T_{F}^{ \pm} & =j^{ \pm} e^{ \pm i(\sqrt{(k+2) / 2}-1) \xi_{R}} \\
& =\psi_{1}^{ \pm} e^{ \pm i(\sqrt{(k+2) / 2}) \xi_{R}}
\end{aligned}
$$

with $\psi_{1}^{ \pm}$being the parafermionic currents. The $N=2$ algebra appears because the non-anomalous combination of $\lambda_{R}$ and $\lambda_{L}$ chiral rotations acts on the supercharge.

We must see whether these currents survive the projection. Level matching is now more intricate: there are four sectors, $N S_{ \pm}$and $R_{ \pm}$, on both the left- and right-moving sides, defined by the fermion numbers

$$
\begin{aligned}
N S_{R+}: & F_{R} \in 2 \mathbf{Z} \\
N S_{R-}: & F_{R} \in 2 \mathbf{Z}+1 \\
R_{R \pm}: & F_{R} \in 2 \mathbf{Z} \pm \frac{1}{2}
\end{aligned}
$$


and correspondingly for $F_{L}$ on the left. Conservation of $F_{R}$ and $F_{L}$ implies the correct fusion $N S \cdot N S=N S, R \cdot R=N S, R \cdot N S=R$. In order that the full world-sheet theory satisfy level matching, a state in a sector $\left(F_{L}, F_{R}\right)$ of the monopole CFT must have spin

$$
L_{0}-\tilde{L}_{0} \in \frac{F_{R}^{2}-F_{L}^{2}}{2}+\mathbf{Z}
$$

For example, the sectors $N S_{L+} R_{R \pm}$ have spin $\frac{1}{8}$ and the sector $N S_{L+} N S_{R-}$ $\operatorname{spin} \frac{1}{2}$.

Note first that in the diagonal sectors (same on right and left), the spin is an integer. From the previous section, the condition for a nontrivial spectrum is $k=N N^{\prime}$. Then if we define $F_{L, R}$ as in eq. (5.12) we have

$$
L_{0}-\tilde{L}_{0}=\frac{F_{R}^{2}-F_{L}^{2}}{2}+|K|-|\tilde{K}| \bmod \mathbf{Z}
$$

which is precisely the correct level mismatch.

Inverting (5.12) gives

$$
\begin{aligned}
J_{3} & =Q_{R} F_{R}+Q_{L} F_{L} \\
J_{3}^{\prime} & =Q_{L} F_{R}+Q_{R} F_{L} .
\end{aligned}
$$

Now, the supercurrent has $J_{3}= \pm 1$ and $J_{3}^{\prime}= \pm \sqrt{\left(N N^{\prime}+2\right) / 2}$. Taking the product of eqs. (5.12) implies $F_{R}^{2}-F_{L}^{2}=1$. If the supercurrent is to appear in the operator algebra (6.3) it therefore must have $F_{R}= \pm 1$ and $F_{L}=0$. Then (6.6) with $J_{3}= \pm 1$ implies $Q_{R}= \pm 1$ as the condition for the supercurrent to appear in the algebra, as expected; we are free to take $Q_{R}=1$ by $\psi_{R} \leftrightarrow \bar{\psi}_{R}$.

There are several special cases. If $Q_{L}=0$ we have a neutral remnant; however, this case requires special treatment because $Q_{L}<Q_{R}$. If $Q_{L}=1$ (or equivalently -1 ), the level $k$ vanishes and the CFT degenerates. We will discuss these cases separately below, after discussing some general issues for $Q_{L} \geq 2$ : spacetime supersymmetry, stability, the connection to the asymptotic spacetime, and fermion zero modes.

In order to have spacetime supersymmetry, we need a weight $\left(0, \frac{1}{8}\right)$ field in the sector $F_{L}=0, F_{R}= \pm \frac{1}{2}$. This would combine with the $x t \times$ internal $\times$ 
ghost spin field $S \Sigma e^{-\varphi / 2}$ of weight $\left(0, \frac{7}{8}\right)$ to produce the $(0,1)$ current corresponding to spacetime supersymmetry. This field can only be $e^{i \xi_{R} \sqrt{k / 8}}$, with $J_{3}=0$ and $J_{3}^{\prime}=\sqrt{k / 8}$. Inserting into $(5.12$ ) gives

$$
F_{R}+F_{L}=\frac{1}{2} \sqrt{\frac{Q_{-}}{Q_{+}}},
$$

which is never a half-integer. Thus these theories are not spacetime supersymmetric, in agreement with the result of the $\alpha^{\prime}$ expansion [3].

Without spacetime supersymmetry, there is the possibility of tachyons. To get the mass-shell condition, we need to look at the $x t$ CFT. In the throat limit the $x t$ energy-momentum tensor is

$$
\frac{1}{2}\left(\partial_{z} t \partial_{z} t+\psi_{t} \partial_{z} \psi_{t}\right)-\frac{1}{2}\left(\partial_{z} x \partial_{z} x+\psi_{x} \partial_{z} \psi_{x}\right)-\alpha \partial_{z}^{2} x .
$$

The total four-dimensional central charge is

$$
3+12 \alpha^{2}+\frac{3 k}{k+2}=6,
$$

SO

$$
\alpha=\frac{1}{\sqrt{2 k+4}}=\frac{1}{2 Q_{L}} .
$$

The primary field

$$
e^{-\alpha x} e^{i k \cdot Y}
$$

with $Y^{\mu}=t, x$ has weight

$$
L_{0}=\frac{k_{\mu} k^{\mu}}{2}+\frac{\alpha^{2}}{2}
$$

A scalar in the -1 picture has total weights $\left(1, \frac{1}{2}\right)$. Using eqs. (5.5) and (6.12), the mass shell condition is

$$
\begin{aligned}
\frac{1}{2} & =\frac{k^{2}}{2}+\frac{(2 j+1)^{2}}{8 Q_{L}^{2}}+\frac{F_{R}^{2}-F_{L}^{2}}{2}+|K|+L_{0}^{\prime}, \\
1 & =\frac{k^{2}}{2}+\frac{(2 j+1)^{2}}{8 Q_{L}^{2}}+|\tilde{K}|+\tilde{L}_{0}^{\prime},
\end{aligned}
$$


where $L_{0}^{\prime}, \tilde{L}_{0}^{\prime}$ are the weights from the internal CFT, plus the contribution of any excitations from the $x t$ theory. To have a solution with $k^{2}>0$, it is necessary that $|K|=0$. The quantum number $J_{3}$ then comes entirely from the primary field, so in particular

$$
2 j+1 \geq 2\left|J_{3}\right|+1=2\left|Q_{R} F_{R}+Q_{L} F_{L}\right|+1 \text {. }
$$

The only possibilities for a tachyon are then円

$$
\begin{array}{ll}
F_{R}=-1, \quad F_{L}=1, & j=Q_{L}-1, \\
F_{R}=-1, & F_{L}=\frac{1}{2}, \quad j=\frac{1}{2} Q_{L}-1 .
\end{array}
$$

For these,

$$
\mu^{2}=-k^{2}=-\frac{\left|F_{L}\right|}{Q_{L}}+\frac{1}{4 Q_{L}^{2}}+2 L_{0}^{\prime}
$$

The operators are

$$
e^{-\alpha x} e^{i k \cdot Y} \tilde{\phi}_{m}^{j} \phi_{j}^{j} e^{-2 i j \xi_{R}} \mathcal{V}_{\text {int }}
$$

for the appropriate $j$ value $(6.15)$. We can use the bosonization formulae from section 4 to to express the result in terms of the original Fermi fields, giving respectively

$$
\begin{array}{r}
e^{-\alpha x} e^{i k \cdot Y} D_{m, j}^{j}\left(g_{\theta, \phi}\right) \lambda_{L} \bar{\lambda}_{R} \mathcal{V}_{\mathrm{int}} \\
e^{-\alpha x} e^{i k \cdot Y} D_{m, j}^{j}\left(g_{\theta, \phi}\right) R_{L+} \bar{\lambda}_{R} \mathcal{V}_{\mathrm{int}} .
\end{array}
$$

The internal part of the vertex operator must have spin $-\frac{1}{2}$ or $-\frac{7}{8}$ respectively in order that the whole operator be the correct $\left(1, \frac{1}{2}\right)$. The simplest case would be a $\left(\frac{1}{2}, 0\right)$ field $\lambda_{L}^{A}$ from the $N S$ sector of the current algebra or a $\left(\frac{7}{8}, 0\right)$ field $R_{L}^{B}$ from the Ramond sector, for which the masses-squared (6.16) will indeed be negative. These vertex operators correspond to gauge bosons, and the current $\lambda_{L}^{A} \lambda_{L}$ and $R_{L}^{B} R_{L+}$ are charged under the $U(1)$ of the monopole.

\footnotetext{
${ }^{1}$ Of course, the could also be the unit operator from the sector $N S_{+}$paired with a state from the sector $N S_{-}$of the internal theory. Such a state, if tachyonic, would also be tachyonic in flat space. Since the theory is supersymmetric in flat space, this is not possible, and we can restrict attention to the sector $N S_{-}$of the monopole theory.
} 
In other words, these states are only present if the $U(1)$ is embedded in an unbroken non-Abelian group in the four-dimensional gauge group. This instability is precisely the Brandt-Neri instability 19] to emission of non-abelian radiation; in field theory language it arises due to the negative centrifugal potential at $j=Q_{L} F_{L}-1$. Even in a theory in which the $U(1)$ is embedded in a broken non-Abelian group the black hole is destabilized if the radius $r_{2}$ of the black-hole throat is small as compared to the breaking scale. The unstable mode produces a non-Abelian monopole core outside the black hole horizon. This is similar to the instability previously studied by Lee, Nair, and Weinberg 20.

Aside from the non-abelian instabilities we have therefore demonstrated the stability of the horizon and throat regions of the magnetic black holes under linearized perturbations.

The vertex operators corresponding to changes in the radii $r_{1}$ and $r_{2}$ are both of the form

$$
\left(\tilde{\jmath}_{-1}^{a} \cdot \tilde{\phi}_{a}^{1}\right)\left(j_{-1}^{b} \cdot \phi_{c}^{1}\right) .
$$

To see this, translate into the WZW fields [11, 14]

$$
\operatorname{tr}\left(g^{-1} \sigma^{a} \partial_{\bar{z}} g\right) \operatorname{tr}\left(\sigma^{b} g^{-1} \partial_{z} g\right) \operatorname{tr}\left(g^{-1} \sigma^{a} g \sigma^{c}\right)=2 \operatorname{tr}\left(\sigma^{b} g^{-1} \partial_{z} g\right) \operatorname{tr}\left(g^{-1} \partial_{\bar{z}} g \sigma^{c}\right) .
$$

Note in particular that this preserves rotational invariance, $S U(2)_{L}$. Now, setting $b=c$ and summing yields $\operatorname{tr}\left(g^{-1} \partial_{z} g g^{-1} \partial_{\bar{z}} g\right)$ giving an overall change in the radius of the WZW model, $\delta r_{1} / r_{1}=\delta r_{2} / r_{2}$. Setting $b=c=3$ changes only the radius $r_{1}$. In a bosonic theory, these are both allowed vertex operators, but in the supersymmetric case we know that supersymmetry relates $r_{1}$ and $r_{2}$, so only one linear combination can be the highest operator in a superfield. The relevant superconformal primary is

$$
\left(\tilde{j}_{-1}^{a} \cdot \tilde{\phi}_{a}^{1}\right)\left(\phi_{+}^{1} e^{i\left(Q_{L}-1\right) \xi_{R}}+\phi_{-} e^{-i\left(Q_{L}-1\right) \xi_{R}}\right) .
$$

Taking the operator product with the supercurrent (6.2) yields the 0-picture vertex operator

$$
\left(\tilde{j}_{-1}^{a} \cdot \tilde{\phi}_{a}^{1}\right)\left(j_{-1}^{+} \cdot \phi_{-}^{1}+j_{-1}^{-} \cdot \phi_{+}^{1}\right)
$$


The primary is irrelevant, has weight

$$
\left(1+\frac{1}{Q_{L}^{2}}, \frac{1}{2}+\frac{1}{Q_{L}^{2}}\right),
$$

so we can make an on-shell primary by multiplying by $e^{x / Q_{L}}$. This perturbation represents the widening of the throat as $x \rightarrow \infty$, but we do not know of an exact CFT which interpolates between the throat and asymptotic region.

Finally, we look for fermion zero modes on the throat.? This gives a useful check on the construction, and will aid us in understanding the physics of the $\left|Q_{L}\right|=1$ solution. A massless fermion of charge $e$ has $2 e Q$ zero modes, by the index theorem. The state

$$
R_{R \pm} \lambda_{L} S_{ \pm} \mathcal{U}_{ \pm} e^{-\varphi / 2}
$$

is a massless fermion in four dimensions, where $S_{ \pm}$is the $\left(0, \frac{1}{8}\right)$ spin field from the $x t$ theory, $\mathcal{U}_{ \pm}$is a $\left(\frac{1}{2}, \frac{3}{8}\right)$ field from the internal theory, $\varphi$ is the bosonized ghost, and $R_{R \pm}$ are the Ramond ground states from the monopole CFT. Its $U(1)$ charge is $e=1$ from $\lambda_{L}$. The appropriate spatial wavefunction is $e^{-\alpha x} e^{i k \cdot Y} D_{m, Q_{L} \pm 1 / 2}^{j}\left(g_{\theta, \phi}\right)$. The mass shell condition $L_{0}=1$ is then

$$
-k^{2}=\frac{(2 j+1)^{2}}{4 Q_{L}^{2}}-F_{L}^{2}
$$

with $F_{L}=1$. Recalling that $j \geq q / 2$, the lowest state is $R_{R-} \lambda_{L}$ at $j=Q_{L}-\frac{1}{2}$. This is indeed massless, and its multiplicity is $2 j+1=2 Q_{L}$ as expected, This can also be phrased as an index. The spectrum is

$$
\begin{array}{ll}
R_{R+}: & j=\quad Q_{L}+\frac{1}{2}, \quad Q_{L}+\frac{3}{2}, \ldots \\
R_{R-}: & j=Q_{L}-\frac{1}{2}, Q_{L}+\frac{1}{2}, Q_{L}+\frac{3}{2}, \ldots
\end{array}
$$

The Ramond generator $G_{0}$ takes $R_{R+} \leftrightarrow R_{R-}$ and anticommutes with $(-1)^{F_{R}}$. We see that all the massive states are appropriately paired, while the massless states are the kernel of $G_{0}$. Another example is

$$
R_{R \pm} R_{L+} S_{ \pm} \mathcal{U}_{ \pm}^{\prime} e^{-\varphi / 2},
$$

\footnotetext{
${ }^{2}$ We thank Tom Banks for suggesting this.
} 
which for $\mathcal{U}_{ \pm}^{\prime}$ of weight $\left(\frac{7}{8}, \frac{3}{8}\right)$ is a massless fermion of charge $e=\frac{1}{2}$. The mass shell condition is again (6.25), with $F_{L}=\frac{1}{2}$, and $R_{R-} R_{L+}$ at $j=\frac{1}{2} Q_{L}-\frac{1}{2}$ indeed gives a massless state of multiplicity $Q_{L}$.

\section{The Neutral Remnant: $Q=0$}

A vanishing monopole field corresponds to $Q_{R}=1, Q_{L}=0$. Since our construction assumes $Q_{R}<Q_{L}$, we need to take the case $Q_{L}=1, Q_{R}=0$ and flip:

$$
\frac{S U(2)_{L} \times S U(2)_{R}}{Z(2)_{L}} .
$$

It might seem strange that there is a neutral solution, since the idea was to balance curvature against magnetic field. In the bosonic form, the curvature is balanced by torsion which arises from the spin connection, both one-loop effects. In the original fermionic variables, a one-loop curvature term is balanced by a two-loop curvature-squared term. Obviously, we cannot be sure from the semiclassical picture that this is possible, but the exact construction shows that it is. The WZW model is known to be conformally invariant, and the $Z(2)$ modding does not affect the $\beta$-functions.

The vertex operators are then the parity transform of the previous discussion,

$$
e^{i w_{3} \zeta_{L}}\left(\tilde{\jmath}_{\tilde{K}} \cdot \tilde{\phi}_{m_{L}}^{j}\right)\left(j_{K} \cdot \phi_{m_{R}}^{j}\right) .
$$

Here, $\zeta_{L}$ is the antianalytic field obtained by bosonizing $U(1)_{L}$, not the same as the earlier $\xi_{L}$ associated with $U(1)_{R}$. The fermion numbers are

$$
\begin{aligned}
& \tilde{J}_{3}=F_{R} \\
& \tilde{J}_{3}^{\prime}=F_{L},
\end{aligned}
$$

where $\tilde{J}_{3}$ is the left $J_{3}$ value of the untwisted operator, and $\tilde{J}_{3}^{\prime}=J_{3}+w_{3}$ is the left $J_{3}$ value of the twisted operator. The spectrum runs over all operators of the form (7.2) with half-integer $F_{R}$ and $F_{L}$.

The $k=2$ current algebra can be represented by a triplet of current algebra fermions, $\psi_{R, L}^{a}$. Note that the original $\lambda_{L}$ are free, because they do 
not couple to the gauge field, and one finds the simple result

$$
\lambda_{L}=e^{i \zeta_{L}}=\psi_{L}^{1}+i \psi_{L}^{2}=\tilde{\phi}_{+}^{1}
$$

Let us check the supercurrent. Taking $\tilde{J}_{3}= \pm 1, \tilde{J}_{3}^{\prime}=0$, one obtains the $\left(0, \frac{3}{2}\right)$ operators

$$
e^{\mp i \zeta_{L}} \tilde{\phi}_{ \pm}^{1} j_{-1}^{a} \cdot \phi_{m}^{1}
$$

In fact, there is only one independent operator here. The left-moving side is the unit operator, as it must be since it has zero weight. The right-hand side is seen from the free fermi representation to be $\delta_{m}^{a}$ times

$$
\psi_{R}^{1} \psi_{R}^{2} \psi_{R}^{3}
$$

the well-known fermionic supercurrent. In this case there is no promotion to $N=2$ : because $Q_{L}=0$, the non-anomalous $U(1)$ acts only on $\lambda_{L}$ and commutes with the supercurrent.

There are no left-moving supersymmetries. Candidates such as $e^{ \pm i \zeta_{L}(\sqrt{2}-1)} \tilde{j}^{ \pm}$ and $\psi_{L}^{1} \psi_{L}^{2} \psi_{L}^{3}$ are not of the form (7.2). The $S U(2)$ rotational symmetry is now on the right-moving side. There is no $S U(2)_{L}$ symmetry. One would have expected this to survive a $Z(2)$ projection, since this is in the center, but the GSO projection uses a $Z(4)$ twist and eliminates the $S U(2)_{L}$ generators.

Again there is no spacetime supersymmetry: $L_{0}=j(j+1) / 4$ cannot take the value $\frac{1}{8}$. Nor can it take the value $\frac{1}{16}$, as would be needed for a fermion zero mode; this confirms the identification $Q=0$. The solution is stable: other than the tachyon, the lowest weight in the $N S_{R}$ spectrum is $\frac{1}{2}$.

There is a technical subtlety, though it presumably does not affect the physics in the end. We believe that this neutral remnant can join smoothly to an external asymptotically flat spacetime, although we cannot construct the exact solution. We should expect, however, to be able to identify the operators in the CFT corresponding to the perturbative broadening of the throat, as in the previous section. The vertex operator (6.19) from the previous section reduces for $k=2$ to

$$
\delta_{c}^{b} \psi_{L}^{1} \psi_{L}^{2} \psi_{L}^{3} \psi_{R}^{1} \psi_{R}^{2} \psi_{R}^{3}
$$


There is only one independent operator, whereas we have two radii. Moreover, this is not the operator we want. It is not the top state in a world-sheet superfield. Also, it preserves the full $S U(2)_{L} \times S U(2)_{R}$ symmetry of the action, whereas the supersymmetric deformation affects only $r_{2}$, since the Thirring interaction vanishes when $Q_{L}=0$.

One might be tempted to assume that there is no vertex operator for deformation of $r_{2}$ (so that this neutral throat could not join to an asymptotic spacetime). This is surely not the case. Over some finite region of $\left(r_{1}, r_{2}\right)$ parameter space, the $\left(Q_{L}, Q_{R}\right)=(0,1)$ sigma model must flow to the WZW fixed point. In particular, start near near the $S U(2) \times S U(2)$ line $r_{1}=r_{2}$; this line is attractive, and on the line we flow to the WZW point. So the WZW theory must have separate moduli for deformation of $r_{1}$ and $r_{2}$. The problem is that at small $k$, the identification of sigma model operators in the CFT becomes more complicated. For example, the wavefunction $D_{m, m^{\prime}}^{j}(g)$ corresponds to a primary only for $k \geq 2 j$. So we need to look for the necessary vertex operator. It has several identifying properties: a scalar under $S U(2)_{R}$, a tensor under $S U(2)_{L}$, independent of $\psi_{L}^{1,2}$ since these are always free, and invariant under world-sheet supersymmetry. An operator with these properties first appears at level $(2,2)$ :

$$
\psi_{L}^{3} \partial_{\bar{z}} \psi_{L}^{3} \sum_{a=1}^{3} \psi_{R}^{a} \partial_{z} \psi_{R}^{a} .
$$

This is globally supersymmetric (its world-sheet integral is annihilated by $\left.G_{-1 / 2}\right)$ and becomes locally supersymmetric and $(1,1)$ with Liouville dressing.

\section{The Degenerate Remnants: $Q= \pm 1$}

For $Q_{L}=Q_{R}=1$, the level $k=2 Q_{+} Q_{-}$vanishes. In the bosonized form, there is no torsion due to the left-right symmetry, and so no nontrivial fixed point. One might conclude that there are no $Q=1$ remnants of this form. However, we would like to suggest a more interesting possibility. Namely, that there is a throat, in which the angular CFT has collapsed to the trivial $k=c=0$ theory, leaving only the the $x t$ theory and the internal theory. 
One way to motivate this is via the renormalization group. Roughly speaking, we would like to think about the radial dependence as a renormalization group flow. For example, in the solutions found thus far, if we have an asymptotically flat external spacetime connected to the throat, then $r_{2}$ flows from $\infty$ to the WZW fixed point value. This identification of radius with world-sheet scale is quantitatively accurate near the WZW fixed point (i.e. in the throat region), because the radial "dressing" needed to convert the kinetic term for the angular modes to a $(1,1)$ operator is near to the identity. However near and outside of the mouth this is no longer the case. It would be quantitatively accurate along the entire flow if the dilaton had a large (in string units) radial derivative. When the dilaton has a radial gradient, the radial coordinate has a classical scale transformation

$$
\delta x=\delta \epsilon G^{x x} \partial_{x} \Phi
$$

so that the radius would approximate a classical Liouville field if the gradient were large. In the present case, the derivative is of order one in string units, so we must hope that the renormalization group picture is a reasonable qualitative guide. Similar ideas have recently been discussed by Polyakov [16], in a cosmological context, and Banks[17], in the context of Schwarzchild black holes.

At very large radius, the complete theory can presumably be analyzed in a sigma-model expansion in $\frac{1}{r_{2}}$. This is equivalent to perturbatively solving the low-energy field equations. One finds that the geometry is asymptotically flat, and that to evolve to smaller $r_{2}$ one must specify as initial data the mass $M$ and the charge $Q .1$ Evolving inward, one eventually reaches the region where $r_{2}$ is of order one and (if $Q$ is also order one) geometric curvatures become of order one and sigma-model perturbation theory breaks down. For generic values of $M$ and $Q$ one expects to encounter a singularity inside this region - one certainly hopes that there are no smooth, negative-mass solu-

\footnotetext{
${ }^{1}$ We consider here theories for which the coefficient of $\partial_{+} t \partial_{-} t$ is independent of $r_{2}$; this excludes non-extremal black holes, and leads to the constraint $M^{2}-2 M D-D^{2}+$ $Q^{2} / 2=0$. More generally the dilaton charge $D$ is required as additional initial data. Our normalization of $\mathrm{D}$ is such that the force between two black holes is proportional to $\left(M_{1} M_{2}+D_{1} D_{2}-Q_{1} Q_{2} / 2\right)$.
} 
tions of string theory! However by tuning $M$ relative to $Q$, it may be possible to flow into a throat region, in which the theory may be approximately analyzed in terms of radial-renormalization group flows of the angular theory on $S_{2}$. This is certainly the case for large $Q$, and in the following we assume that it is also true for $Q=1$.

The $Q=1$ monopole theory is a nonlinear sigma model without torsion. It is thus expected to flow to strong coupling and develop a mass gap, so the low energy theory is the trivial $c=0 \mathrm{CFT}$. This is consistent with studies of this particular model[15], which is also (in fermionic form) the $(2,2)$ supersymmetric $O(3)$ nonlinear sigma model. Applying this to the black hole, the sigma model would become strongly coupled in the mouth region, and in the throat only the $c=0$ theory would survive, as suggested above.

Let us consider the spectrum of the string theory in the throat. The sigma model has a $Z(2)$ chiral symmetry

$$
\lambda_{L} \rightarrow-\lambda_{L}, \quad \lambda_{R} \rightarrow \lambda_{R}
$$

which is non-anomalous: the instanton amplitude from $S_{2} \rightarrow S_{2}$ with unit winding is of the form $\lambda_{L}^{2 Q_{L}} \bar{\lambda}_{R}^{2}$. This $Z(2)$ is broken at strong coupling 15,

$$
<\lambda_{L} \bar{\lambda}_{R}>\propto \pm 1
$$

At low energy - that is, in the throat - the characteristic length scale of the sigma model is large compared to the size of the string. There are therefore two ground states. To be precise, there are two such vacua, $| \pm\rangle_{R R}$ (eigenstates of the order parameter (8.3) ), in the purely periodic $R_{L} R_{R}$ sector. Both of these are supersymmetric, as we will see explicitly below from an index theorem. Fermion number acts on these as

$$
(-1)^{F_{R}}| \pm\rangle_{R R}=i|\mp\rangle_{R R}, \quad(-1)^{F_{L}}| \pm\rangle_{R R}=-i|\mp\rangle_{R R}
$$

These are determined, up to redefinition, by the requirement that $(-1)^{F_{R, L}}$ square to -1 in the Ramond sector, and by the requirement that $(-1)^{F_{R}+F_{L}}$, being part of a continuous $U(1)$ symmetry, leave the ground state invariant. 
The fermion number eigenstates are

$$
|+\rangle_{R R} \pm|-\rangle_{R R}, \quad(-1)^{F_{R}}=-(-1)^{F_{L}}= \pm i
$$

In the $R_{L} N S_{R}$ and $N S_{L} R_{R}$ sectors the order parameter $\lambda_{L} \bar{\lambda}_{R}$ is antiperiodic. All such states must contain a kink, and so are massive. In the $N S_{L} N S_{R}$ sector, there are again two ground states, $| \pm\rangle_{N S N S}$, with

$$
(-1)^{F}| \pm\rangle_{N S N S}=(-1)^{F_{L}}| \pm\rangle_{N S N S}=|\mp\rangle_{N S N S}
$$

The fermion number eigenstates are

$$
|+\rangle_{N S N S} \pm|-\rangle_{N S N S}, \quad(-1)^{F}=(-1)^{F_{L}}= \pm 1 \text {. }
$$

Although supersymmetry is broken in this sector, the effects are exponentially small (in the ratio of the string size to the length scale of the sigma model) due to the mass gap. In the throat region the monopole CFT thus has the four states (8.5) and (8.6), all of essentially zero weight. One usually expects only a single zero-weight state, but these states are distinguished by the massive degrees of freedom.

From the index theorem for spacetime zero modes discussed in section 6 , we expect one state of weight $(0,0)$ in each of the sectors $R_{L+} R_{R-}$ and $R_{L-} R_{R+}$. These we have found, so the throat theory is nontrivial: there are massless fermions. The earlier discussion would also lead us to expect two states of weight $\left(\frac{3}{8}, 0\right)$ in each of the sectors $N S_{L-} R_{R \pm}$. These cannot exist if there is a mass gap: only zero weight is allowed in the trivial CFT. In fact, these states get mass from instantons. The mass in the effective $x t$ Dirac equation for these two states is

$$
\left\langle\bar{\lambda}_{L} R_{R+}\left|G_{0}\right| \lambda_{L} R_{R-}\right\rangle
$$

Precisely for $Q_{L}=1$ the instanton amplitude $\lambda_{L}^{2 Q_{L}} \bar{\lambda}_{R}^{2}$ allows such a matrix element to be nonvanishing.

For completeness let us state the index argument in a careful way. The index is

$$
I=\operatorname{tr}\left(\delta_{L_{0}-\tilde{L}_{0}, s} e^{\pi i F_{L} / Q_{L}+i \pi F_{R}}\right)
$$


the trace being taken in the supersymmetric $R_{R}$ sector. This is $(-1)^{F_{R}}$, restricted to a sector of given spin and weighted by the $U(1)$ charge $F_{L}$ modulo the anomaly. Note that the operator in the trace is a non-anomalous symmetry, and anticommutes with $G_{0}$. We will calculate this in two limits. The first is $r_{2}$ very large. Here, acting on a state whose right-moving part is $R_{R \pm}$ and whose left-moving part has fermion number $F_{L}$, the Ramond generator reduces to the Dirac operator for $e=F_{L}$ on the two-sphere, and so this state should contribute $\mp i 2 Q_{L}\left|F_{L}\right| e^{\pi i F_{L} / Q_{L}}$ to the trace. Thus, we have from the discussion in section 6 ,

$$
\begin{array}{cl}
s=0: & I=2 Q_{L} \sin \left(\pi / 2 Q_{L}\right) \\
s=-\frac{3}{8}: & I=4 Q_{L} \sin \left(\pi / Q_{L}\right) .
\end{array}
$$

For $Q_{L}=1$, the second index vanishes, so there is no contradiction with the spectrum we have found.

The theory is not spacetime supersymmetric; there are no $N S_{L} R_{R}$ states at all in the throat. As before, it is unstable if embedded in a non-Abelian gauge group due to low-lying $N S_{R}$ - states, but becomes stable if the nonAbelian bosons are sufficiently heavy, eq. (6.16) giving $m^{2} \geq 1 / 4$.

The angular dimensions have effectively shrunk to zero radius, leaving behind some massless states but no other angular excitations at all. 9 This is a counterexample to the phenomenon of duality familiar from tori.

\section{Conclusions}

We have studied a string theory with right-moving supersymmetric fermions and left-moving current algebra fermions. There are various generalizations of our construction. For example, supersymmetric magnetic black holes [18, 2] are solutions of string theory when the $U(1)$ charge arises from

\footnotetext{
${ }^{2}$ Another curiousity: regarded as a bosonic compactification, there are effectively two throats. Strings in different chiral vacua (8.3) cannot interact in the throat because the cost would be prohibitive. There are even two non-interacting gravitational fields, and so the picture of two throats appears to be a natural interpretation. In the heterotic theory, the GSO projection always forces a particular linear combination of the two vacua.
} 
toroidal compactification. At least in some cases these can be described beginning from a supersymmetric version of (1.1). Recently there has been some discussion 21] of non-Abelian black holes and monopoles related to compactifications of the symmetric fivebrane 9]. These may also be constructed with left-right symmetric generalizations of our procedure. Finally, by an electromagnetic duality rotation in the effective field theory one may construct dyon solutions 22]. These solutions also have infinite throats like the purely magnetic black holes. Although these non-trivially mix the $x t$ theory with the angular theory, one might hope to describe them by a related construction.

Exact, modular invariant $(0,2)$ CFTs have made only rare appearances in the literature. The examples given here might also be used for $(0,2)$ compactifications of the heterotic string. Indeed, one of the original motivations of this work was a (thus far unsuccessful) search for models with small tree-level supersymmetry breaking.

Potentially the most interesting - and certainly the most tenuous - result of this paper is the stringy resolution of timelike gravitational singularities by an infinitely massive two-dimensional field theory. While only one particular case has been discussed in detail, the idea is clearly very general. We certainly feel that it merits further investigation.

\section{Acknowledgements}

We thank Tom Banks, Mike Douglas, Jeff Harvey, and Steve Shenker for helpful discussions. This work was supported by DOE grant DOE-

91ER40618 and by NSF grants PHY-91-57463, PHY-90-09850, and PHY89-04035. 


\section{Bibliography}

[1] E. Witten, "On string theory and black holes," Phys. Rev. D44 (1991) 314.

[2] G.W. Gibbons and K. Maeda, "Black holes and membranes in higherdimensional theories with dilaton fields,"Nucl. Phys. B298 (1988) 741.

[3] D. Garfinkle, G. Horowitz, and A. Strominger, "Charged black holes in string theory," Phys. Rev. D43 (1991) 3140, erratum Phys. Rev. D45 (1992) 3888.

[4] J.H. Horne and G.T. Horowitz, "Black holes coupled to a massive dilaton," UCSB preprint UCSBTH-92-17, hep-th/9210012.

[5] R. Gregory and J.A. Harvey, "Black holes with a massive dilaton," Phys. Rev. D47 (1993) 2411, hep-th/9209070.

[6] G.T. Horowitz, "The dark side of string theory: black holes and black strings," UCSB preprint UCSBTH-92-32, hep-th/9210119, to appear in the proceedings of the 1992 Trieste spring school on Quantum Gravity and String Theory;

"What is the true description of charged black holes?" UCSB preprint UCSBTH-92-52, gr-qc/9301008.

[7] S.B. Giddings and A. Strominger, "Dynamics of extremal black holes," Phys. Rev. D46 (1992) 627.

[8] G. Mandal, A Sengupta, and S. Wadia, "Classical solutions of twodimensional string theory," Mod. Phys. Lett. A6 (1991) 1685. 
[9] J. A. Harvey, C. G. Callan and A. Strominger, "Worldsheet approach to heterotic solitons and instantons" Nucl. Phys. B359 (1991) 611.

[10] S.B. Giddings and A. Strominger, "Exact black fivebranes in critical superstring theory," Phys. Rev. Lett. 67 (1991) 2930.

[11] E. Witten, "Non-Abelian bosonization in two dimensions," Comm. Math. Phys. 92 (1984) 455.

[12] S. Mandelstam, "Soliton operators for the quantized Sine-Gordon equation," Phys. Rev. D11 (1975) 3026.

[13] T.T. Wu and C.N. Yang, "Dirac monopole without strings: monopole harmonics," Nuc. Phys. B107 (1976) 365;

"Some properties of monopole harmonics," Phys. Rev. D16 (1977) 1018.

[14] D. Gepner and E. Witten, "String theory on group manifolds," Nucl. Phys. B278 (1986) 493.

[15] E. Witten, Nucl. Phys. B149 (1979) 285;

A. D’Adda, P. Di Vecchia, and M. Luscher, Nucl. Phys. B152 (1979) 125.

[16] A. M. Polyakov, "A few projects in string theory", to appear in the proceedings of the 1992 Les Houches Summer School.

[17] T. Banks, unpublished.

[18] G. W. Gibbons and C. M. Hull, Phys. Lett. 109B (1992) 190;

R. Kallosh, A. Linde, T. Ortin, A. Peet and A. Van Proeyen, Stanford preprint hep-th/920507, to appear Phys. Rev. D;

T. Ortin, Stanford preprint hep-th/9208078, SU-ITP-92-24.

[19] R.A. Brandt and F. Neri, "Stability analysis for singular nonabelian magnetic monopoles," Nucl. Phys. B161 (1979) 253. 
[20] K. Lee, V.P. Nair, and E.J. Weinberg, " A classical instability of Reissner-Nordstrom solutions and the fate of magnetically charged black holes," Phys. Rev. Lett. 68 (92) 1100, hep-th/9111045; "Black holes in magnetic monopoles," Phys. Rev. D45 (1992) 2751, hep-th/9112008.

[21] J. A. Harvey and J. T. Liu, Phys. Lett. B268 (1991) 40;

J. P. Gauntlett, J. A. Harvey and J. T. Liu, EFI preprint EFI-92-67;

R. R. Khuri, Texas A\&M preprints CTP-TAMU-33/92 (hepth/9205051); CTP-TAMU-35/92 (hep-th/9205081).

[22] A. Shapere, S. Trivedi, F. Wilczek, "Dual dilaton dyons," Mod. Phys. Lett. A6 (1991) 2677. 